\title{
Toints
}

$\sqrt{2}$

\section{Analysis of differences in bone removal during femoral box osteotomy for primary total knee arthroplasty}

\author{
ANGELO GRACEFFA ${ }^{1}$, PIER FRANCESCO INDELLI ${ }^{2,3,4}$, KAITLYN BASNETT³, \\ MASSIMILIANO MARCUCCI ${ }^{2,4}$ \\ ${ }^{1}$ Orthopaedics Clinic, University of Catania, Italy \\ ${ }^{2}$ Centro Eccellenza Sostituzioni Articolari Toscana (CESAT), Orthopaedics Clinic, University of Florence, Italy - \\ Fondazione Onlus “...In Cammino...”, Fucecchio, Italy \\ ${ }^{3}$ Breyer Center for Overseas Study, Stanford University in Florence, Italy \\ ${ }^{4}$ Orthopaedics Clinic, University of Florence, Italy
}

\begin{abstract}
Purpose: this study was conducted to compare the quantity of intercondylar bone removed during femoral box osteotomy for implantation of three contemporary posterior stabilized (PS) total knee arthroplasty designs: Sigma PS (DePuy), Vanguard (Biomet) and Persona (Zimmer).

Methods: we compared the maximum volumetric bone resection required for the housing of the PS mechanism of these three designs. Bone removal by each PS box cutting jig was three-dimensionally measured. The differences between the three designs were analyzed by the Kruskal-Wallis test. The MannWhitney U-test was used for pairwise comparisons. The level of significance was set at $\mathrm{p}<0.05$.

Results: for small-size implants, the average box osteotomy volume of Persona was significantly smaller than the Vanguard and Sigma PS volumes $(\mathrm{p}=0.003)$. The mean difference between Vanguard and Sigma PS $(\mathrm{p}=0.01)$ was also significant. For medium size implants, the mean difference between Persona and Sigma PS $(\mathrm{p}=0.008)$ and the mean difference between Vanguard and Sigma PS $(\mathrm{p}=0.01)$ were statistically significant. For large size implants, the mean difference between Vanguard and Sigma PS $(\mathrm{p}=0.01)$ and the mean difference between Sigma PS
\end{abstract}

Corresponding Author:

Pier Francesco Indelli, MD

CESAT

Piazza Lavagnini 1, 50054 Fucecchio (Fi), Italy

E-mail: pierfrancesco.indelli@unifi.it and Persona $(\mathrm{p}=0.008)$ were statistically significant. Conclusions: irrespective of implant size, the Persona cutting jig always resected significantly less bone than did Vanguard and Sigma PS.

Clinical Relevance: although this study does not establish any clinical relevance of removing more or less bone at primary TKA, its results suggest that if a PS design is indicated, it is preferable to select a model which resects less distal femoral bone.

Key Words: bone resection, box osteotomy, cruciate substituting, posterior stabilized, total knee arthroplasty.

\section{Introduction}

The use of posterior-stabilized (PS) implants in total knee arthroplasty (TKA) is becoming increasingly popular. Many designs, which are modifications of the original Insall-Burstein prosthesis (Zimmer, Warsaw, IN, USA) (1), have inherited the classical post-andcam mechanism, used in lieu of the posterior cruciate ligament, for guiding anteroposterior knee motion. The potential advantages of PS TKA include the possibility of easier balancing of severe coronal and sagittal deformities (i.e. varus, valgus or recurvatum), better controlled flexion kinematics, less polyethylene sliding wear, greater weight-bearing maximal flexion, and greater posterior femoral roll-back than are observed with cruciate-retaining (CR) high-flexion TKA (2). A reduction of patellofemoral contact pressure in PS TKA designs when compared with CR designs is another potential advantage (3). There are 
several potential disadvantages of PS designs with respect to other CR implants, and these include tibial post wear and breakage (4), increased incidence of anterior knee pain (5), and implant instability, especially during the flexion phase (6).

One of the main disadvantages of PS designs (compared with CR implants) is also linked to the amount of bone removed during femoral osteotomy to create the housing of the PS mechanism (box osteotomy). Theoretically, greater preservation of bone stock would be advisable with a view to possible future revision of the implant.

The purpose of the present study was to compare the quantity of intercondylar bone removed during femoral box osteotomy for implantation of the femoral component of three contemporary posterior PS TKA designs.

The hypothesis of the study was that the maximum volume of bone resection during femoral box osteotomy differs significantly between different PS TKA designs.

\section{Methods}

The complete surgical instrumental sets of the following three PS TKA devices were available for the present study: Sigma PS (De Puy, Johnson and Johnson, Warsaw, IN, USA), Persona (Zimmer, Warsaw, IN, USA) and Vanguard (Biomet Inc, Warsaw, IN, USA). This study excluded the analysis of very small ("micro") and very large ("macro") size implants.

Femoral box osteotomy is a surgical procedure involving application of a cutting jig to the distal part of the femur (Fig. 1). The Sigma PS TKA surgical instrumentation set includes two cutting jigs for small femoral sizes (sizes 2 and 2.5), two jigs for medium femoral sizes (sizes 3 and 4) and one jig for large sizes (size 5). The Vanguard TKA surgical instrumentation set includes three cutting jigs for small femoral sizes (sizes 55, 57.5, 60), three jigs for medium femoral sizes (sizes $62.5,65,67.5$ ) and three jigs for large sizes (sizes 70, 72.5, 75). The Persona TKA surgical instrumentation set includes one cutting jig for small femoral sizes (sizes 3-5), one jig for medium femoral sizes (sizes 6-9) and one jig for large sizes (sizes 10-12).

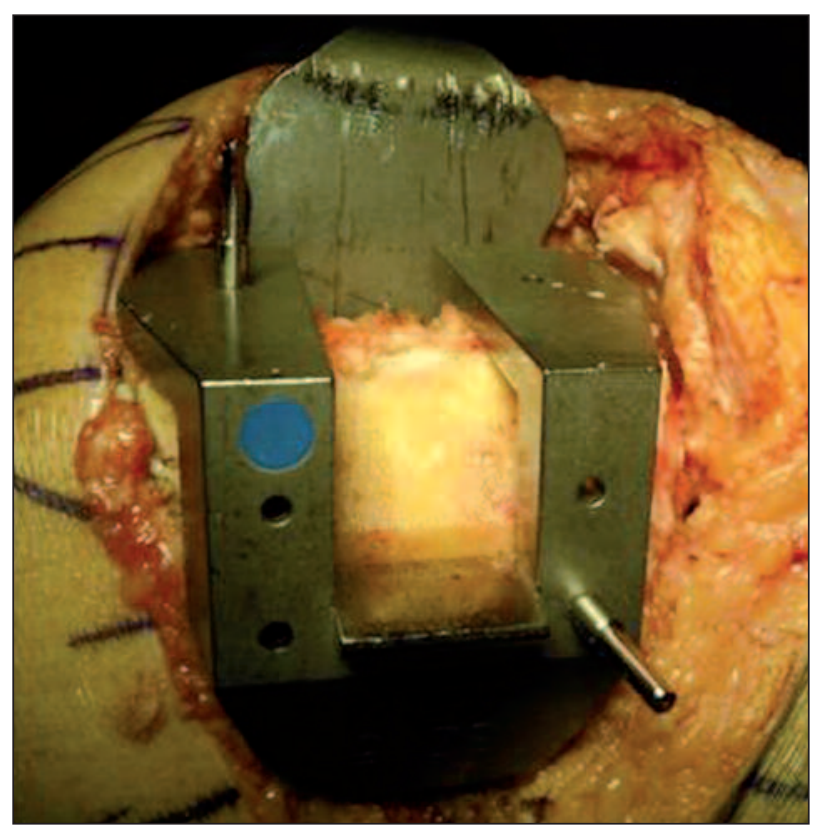

Fig. 1. Intraoperative image of a femoral jig before "box osteotomy" (right knee).

Indirect measurements of removed bone were acquired by measuring the size of the space for the box osteotomy on each cutting jig. Measurements were acquired three times for each sample using a millimeter caliper in an anterior-to-posterior (length), medial-to-lateral (width) and proximal-todistal (depth) direction. Data were collected on an electronic spread sheet. Statistical analysis was performed using the Kruskal-Wallis test for comparison between groups, with box volume as the dependent variable. The Mann-Whitney U-test was used for pair wise comparisons. Significance was set at $p$ $<0.017$ and Bonferroni's correction was applied.

\section{Results}

Analysis of differences between the three implants was significant for all the implant sizes (Fig. 2).

For the small implants, the average box osteotomy volume was $11.04 \mathrm{~cm}^{3}$ for Vanguard, $24.03 \mathrm{~cm}^{3}$ for Sigma PS and $6.18 \mathrm{~cm}^{3}$ for Persona $(\mathrm{p}=0.002)$. Pairwise comparisons showed that the box osteotomy volume of Persona was significantly smaller than those 


\section{j}

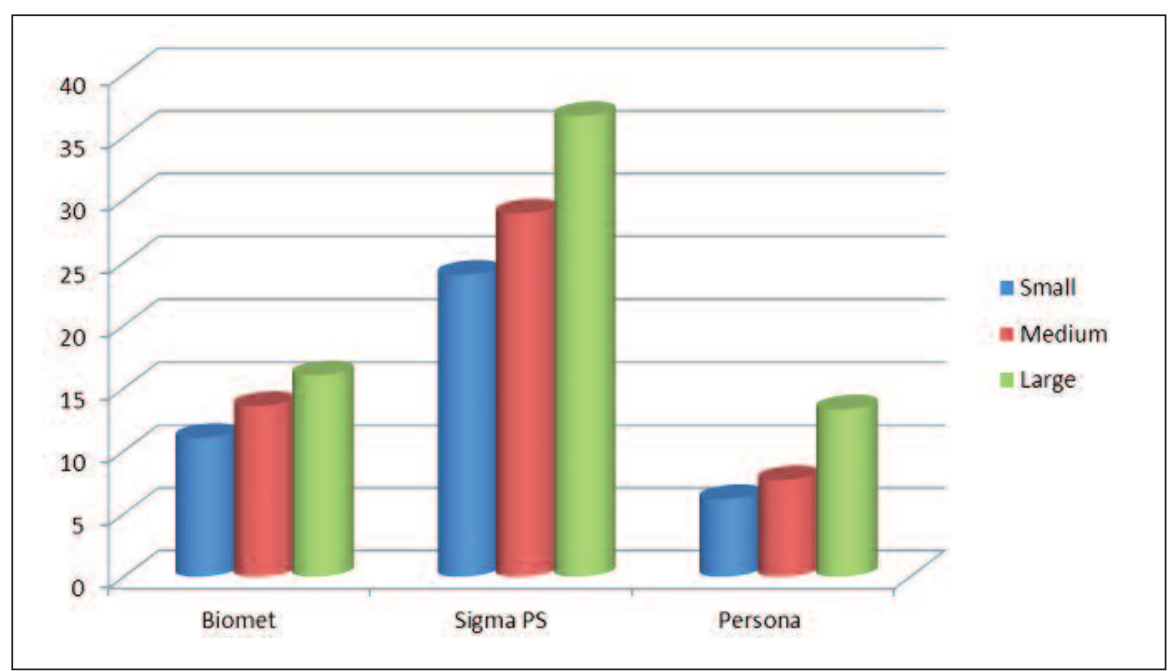

Fig. 2. Bar diagram showing the maximum volumetric bone resection $\left(\mathrm{cm}^{3}\right)$ for each PS cutting jig of the tested designs.

of Vanguard and Sigma PS ( $\mathrm{p}=0.003)$. The difference between Vanguard and Sigma PS $(\mathrm{p}=0.01)$ was also significant.

For medium size implants the average box osteotomy volume was $13.59 \mathrm{~cm}^{3}$ for Vanguard, $28.91 \mathrm{~cm}^{3}$ for Sigma PS and $7.69 \mathrm{~cm}^{3}$ for Persona $(\mathrm{p}=0.003)$. Pairwise comparisons showed that the difference between Persona and Sigma PS ( $\mathrm{p}=0.008)$ and the difference between Vanguard and Sigma PS $(\mathrm{p}=0.01)$ were statistically significant.

For large size implants, the average box osteotomy volume was $16.05 \mathrm{~cm}^{3}$ for Vanguard, $36.66 \mathrm{~cm}^{3}$ for Sigma PS and $13.32 \mathrm{~cm}^{3}$ for Persona $(\mathrm{p}=0.006)$. Pairwise comparisons showed that the difference between Vanguard and Sigma PS ( $\mathrm{p}=0.01)$ and the difference between Sigma PS and Persona $(\mathrm{p}=0.008)$ were statistically significant.

The volumetric bone resection for the PS housing does not include resection for the femoral lugs, typical of the Zimmer Persona design. In fact, with this PS TKA design, an additional $0.59 \mathrm{~cm}^{3}$ of bone was removed for each of the two lugs.

\section{Discussion}

Preservation or substitution of the posterior cruciate ligament in primary TKA is still a controversial issue
(7). Gait analysis, in vivo and in vitro studies showed reproduction of close-to-normal knee kinematics using either solution (8-10). A clinical comparative study between different types of TKA (CR or PS) with identical femoral geometry showed similar mid-term outcomes with regard to range of motion (ROM), functional outcomes and survival rate (11).

The purpose of this study was to compare the maximum quantity of intercondylar bone removed when using three widely used contemporary PS TKA designs. The implants tested in the current study have the characteristic of producing similar kinematics in the PS mechanism, including flexion angle for post-and-cam interaction.

The PFC-Sigma (DePuy) TKA was introduced in 1996 as an improvement of the Press-Fit Condylar (PFC) implant (Johnson \& Johnson, Raynham, Massachusetts, USA). Design features of the PS housing include an updated femoral coronal geometry and a deeper and more prolonged trochlear groove to improve patellar tracking. Recently, a few studies have investigated the functional outcome of the PFC and PFC-Sigma knee systems (12) and found them to show satisfactory mid-term results. Some authors reported minor extensor mechanism complications following the use of this implant (13). Because of these patellofemoral problems, the PFC-Sigma femoral component was re-designed, becoming available in 2009 under the name PFC Sigma PS (DePuy). The principal modifications regarding the PS housing design included a "J curve" femoral design, a new femoral box and smoother trochlear groove edges: these design changes provided a better patellar route during ROM (5). The Sigma PS implant shows an in vivo posterior post-cam engagement at an average of $93^{\circ}$ of flexion (14). A new specific TKA instrumentation (High Performance: HP; DePuy) was introduced as well, including a new PS box cutting jig which was evaluated in the current study (Fig. 3).

The Biomet Vanguard PS TKA was first introduced in 


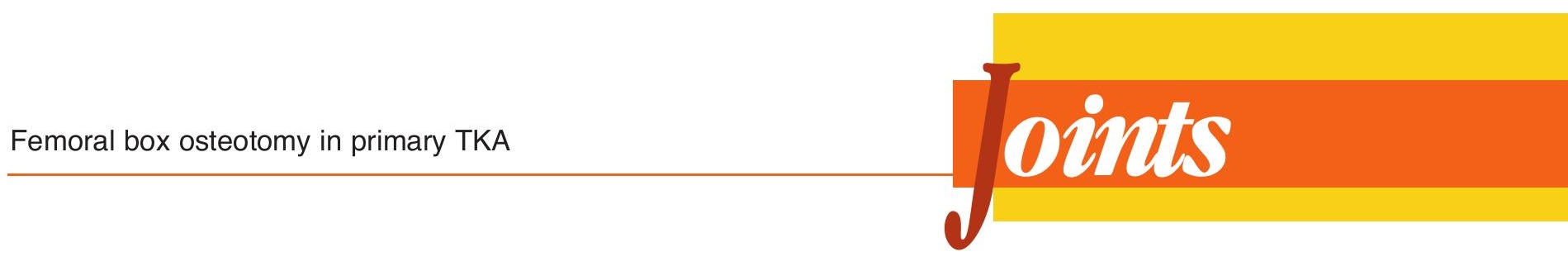

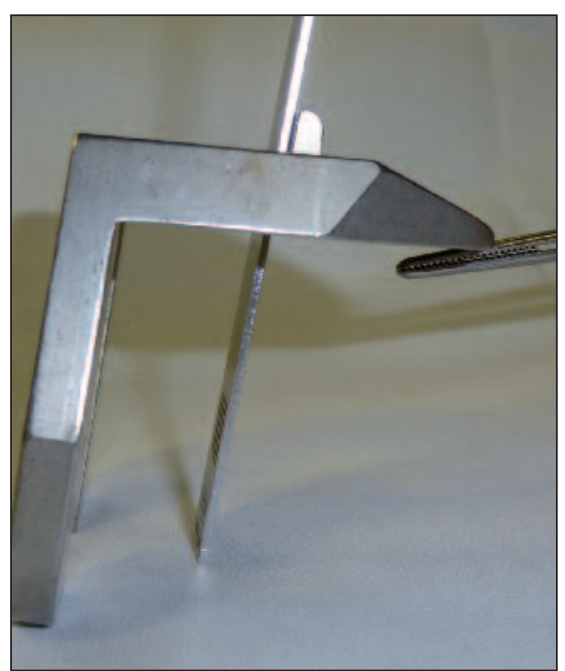

Fig. 3. The Sigma PS (De Puy) femoral cutting jig (large sizes): an osteotome defines area of removable bone.

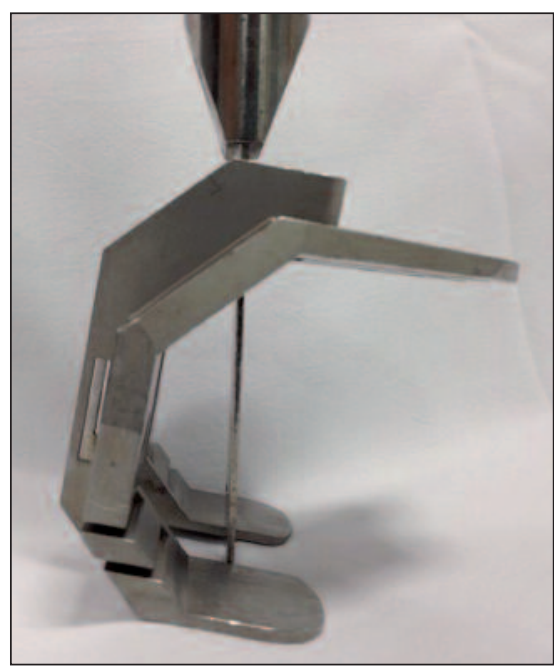

Fig. 4. The Vanguard (Biomet) femoral cutting jig (large sizes): an osteotome defines area o removable bone.

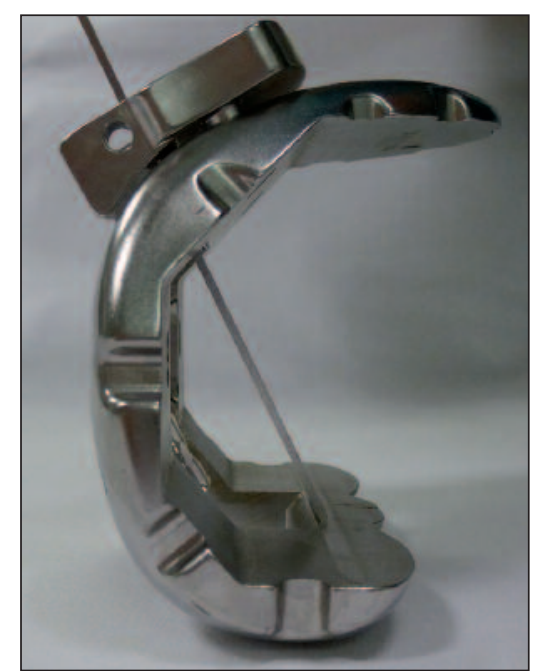

Fig. 5. The Persona (Zimmer) femoral cutting jig (large sizes): an osteotome defines area of removable bone.
2003. The femoral component had a rounded sagittal profile and a wide, deep and long trochlear groove. The standard box resection cut is parallel to the distal femoral resection (Fig. 4). This implant rolls and slides anteriorly until it engages the post-and-cam mechanism at $78^{\circ}$ of flexion. This design has given very promising mid-term clinical results (15).

The Zimmer Persona design was developed from the experience of the Insall-Burstein PS knee (Zimmer), which had a post-and-cam articulation at $60^{\circ}$ of knee flexion and the Nex-Gen Legacy (Zimmer), which showed initial post-cam engagement at $100^{\circ}$ of knee flexion (16). Both of these implants showed satisfactory clinical results $(1,17)$. The Zimmer Persona TKA is characterized by side-specific implants, an increase in size selection having gender-specific measures, anatomical tibial plates, and a femur-shaped cutting jig (Fig. 5).

The surgical instrumentation of these knee systems includes cutting jigs for small, medium and large implants and in the current study these were analyzed separately. The results of the present study showed that the volume of femoral box osteotomy of Persona was significantly smaller than those of the other designs evaluated. These results indicate that TKA designs differ significantly in bone volume resection, some of them requiring over twice as much as bone removal from the intercondylar notch, especially for small and medium implant sizes. Quite apart from need to save bone stock with a view to a possible implant revision, resection of intercondylar bone may create a potential stress rise in the distal femur, predisposing to intercondylar fracture. In this study, we are not attempting to point out a better design at the expense of another one, but simply to identify a less invasive surgical instrumentation for cases in which a PS solution is needed. The post-and-cam mechanisms for these designs have many differences with regard to size, position and articulating geometries.

Very few studies have compared bone loss between different types of PS TKA implants. Haas et al. (18) showed significant differences in bone removal between different PS designs: unfortunately, none of them were modern, "patella-friendly" implants. Recently, Wragg et al. (19) compared the amount of bone removal between PS and CR designs on saw bone models, showing that PS implants require significantly more bone removal.

This study has several limitations. First, our measurements were performed in an in vitro setting. We measured and compared the maximum volume of bone resection required for the PS housings using different PS box cutting jigs and not the actual quantity of bone removed during in vivo box osteotomy. The amount of bone that can be removed in an in vivo scenario is variable and also dependent on the conformation of the 


\section{Toints}

$\sqrt{2}$

femoral condyles and the level of distal femoral resection. Anyway, many intramedullary femoral resection guides provide a standard $10 \mathrm{~mm}$ distal femur osteotomy. Second, the clinical value of removing more or less bone during primary TKA remains to be fully established. Our hypothesisis that resection of a larger amount of bone from the intercondylar notch might have an influence on in vivo knee kinematics, polyethylene wearing, stability and overall survivor ship of the implant. However, this hypothesis could not be tested in the present study.

In conclusion, this study shows clear differences in the bone-sparing characteristics of current PS TKA surgical instrumentations. A clear clinical advantage is still to be proven, but bone stock preservation is fundamental with a view to future revision surgeries and cutting jigs should be designed to preserve as bone stock as possible when a PS solution is chosen.

\section{References}

1. Indelli PF, Aglietti P, Buzzi R, et al. The Insall-Burstein II prosthesis: a 5- to 9-year follow-up study in osteoarthritic knees. J Arthroplasty. 2002;17:544-549.

2. Seon JK, Park JK, Shin YJ, et al. Comparisons of kinematics and range of motion in high-flexion total knee arthroplasty: cruciate retaining vs. substituting designs. Knee Surg Sports Traumatol Arthrosc. 2011;19:2016-2022.

3. Becher C, Heyse TJ, Kron N, et al. Posterior stabilized TKA reduce patellofemoral contact pressure compared with cruciate retaining TKA in vitro. Knee Surg Sports Traumatol Arthrosc. 2009;17:1159-1165.

4. Puloski SK, McCalden RW, MacDonald SJ, et al. Tibial post wear in posterior stabilized total knee arthroplasty. An unrecognized source of polyethylene debris. J Bone Joint Surg Am. 2001;83-A:390-397.

5. Indelli PF, Marcucci M, Pipino G, et al. The effects of femoral component design on the patello-femoral joint in a PS total knee arthroplasty. Arch Orthop Trauma Surg. 2014;134:59-64.

6. Rodriguez-Merchan EC. Instability following total knee arthroplasty. HSS J. 2011;7:273-278.

7. Pagnano MW, Cushner FD, Scott WN. The role of the posterior cruciate ligament in total knee arthroplasty. J Am Acad Orthop Surg. 1998;6:176-187.

8. Joglekar S, Gioe TJ, Yoon P, et al. Gait analysis comparison of cruciate retaining and substituting TKA following PCL sacrifice. Knee. 2012;19:279-285.

9. Stiehl JB, Dennis DA, Komistek RD, et al. In vivo kinematic comparison of posterior cruciate ligament retention or sacrifice with a mobile bearing total knee arthroplasty. Am J Knee Surg. 2000;13:13-18.

10. Li G, Zayontz S, Most E, et al. Cruciate-retaining and cruciate-substituting total knee arthroplasty: an in vitro comparison of the kinematics under muscle loads. J Arthroplasty. 2001;16(Suppl 1):150-156.

11. Lee SM, Seong SC, Lee S, et al. Outcomes of the different types of total knee arthroplasty with the identical femoral geometry. Knee Surg Relat Res. 2012;24:214-220.

12. Hanusch B, Lou TN, Warriner G, et al. Functional outcome of PFC Sigma fixed and rotating-platform total knee arthroplasty. A prospective randomized controlled trial. Int Orthop. 2010; 34:349-354.

13. Ranawat AS, Ranawat CS, Slamin JE, et al. Patellar crepitation in the P.F.C. sigma total knee system. Orthopedics. 2006;29 (9 Suppl):S68-70.

14. Zingde SM, Leszko F, Sharma A, et al. In vivo determination of cam-post engagement in fixed and mobile-bearing TKA. Clin Orthop Relat Res. 2014;472:254-262.

15. Kievit AJ, Schafroth MU, Blankevoort L, et al. Early experience with the Vanguard complete total knee system: 2-7 years of follow-up and risk factors for revision. J Arthroplasty. 2014;29:348-354.

16. Moynihan A, Varadarajan K, Hanson GR, et al. In vivo knee kinematics during high flexion after a posterior-substituting total knee arthroplasty. Int Orthop. 2010;34:497-503.

17. Zimlitski M, Bhokray KK, Rajan BM, et al. Total knee arthroplasty with NexGen legacy posterior stabilized. Georgian Med News. 2011;(199):14-20.

18. Haas SB, Nelson CL, Laskin RS. Posterior stabilized knee arthroplasty: an assessment of bone resection. Knee. 2000;7:25-29.

19. Wragg R, Khan RJZ, Damasena ITW, et al. A comparison of bone loss at total knee replacement: posterior stabilized versus cruciate retaining. J Orthopaedics. 2012;9:e1. 